Obesity is a new risk factor, to which more and more research is devoted, related to the development of cancer. Many studies of recent years have drawn attention to the role of adipose tissue as an important internal endocrine organ. In the adipose tissue proteins are produced, referred to by the common name as adipokines. In the case of obesity, the neoplasm cells are constantly stimulated by pro-inflammatory cytokines and adipokines, among which leptin dominates. The studies show that leptin can affect the cancer cells through numerous phenomena, e.g. inflammation, cell proliferation, suppression of apoptosis and angiogenesis. In this literature review we examined the role of leptin in the development of the individual cancers: breast cancer, colorectal cancer, prostate cancer, ovarian cancer, endometrial cancer and brain neoplasms: glioma and meningioma. However, leptin has very complicated mechanisms of action which require better understanding in certain types of cancer.

Key words: leptin, obesity, cancer, carcinogenesis.

Contemp Oncol (Pozn) 2019; 23 (2): 63-68 DOI:https://doi.org/10.5114/wo.2019.85877

\section{The influence of leptin on the process of carcinogenesis}

\author{
Patrycja Modzelewska ${ }^{1,2}$, Sylwia Chludzińska ${ }^{1,2}$, Jolanta Lewko', \\ Joanna Reszeć ${ }^{2}$
}

${ }^{1}$ Department of Integrated Medical Care, Medical University of Bialystok, Poland ${ }^{2}$ Department of Medical Pathomorphology, Medical University of Bialystok, Poland

\section{Introduction}

Obesity is a new risk factor, which is the focus of more and more research related to the development of cancer. In the last decade, many research results seem to confirm the influence of obesity on the increase in cancer incidence and mortality. The International Agency for Research on Cancer states that there is sufficient evidence of an association with excess adipose tissue, including overweight and obesity, with occurrence of at least 13 cancers. The spectrum of these cancers includes cancers of the esophagus, breast, colon and rectum, endometrium, gall bladder, stomach, kidney, liver, ovary, pancreas and meningioma [1]. Many studies of the recent years draw attention to the role of adipose tissue as an important internal endocrine organ. In the adipose tissue proteins are produced, referred to by the common name as adipokines. These include, among other things, leptin [2]. Leptin (Ob/LEP) is a product of the obesity gene is located on chromosome 7. It plays a key role as a hormone regulating body mass homeostasis, by lowering appetite. This regulation relies on suppressing the synthesis of neuropeptide hypothalamic $Y$, which is the main stimulator of food intake. By controlling appetite and energy expenditure in response to a change in body weight, leptin plays the role of a specific lipostat in the body. In obese people there is a high level of leptin in serum, which is directly correlated with the total amount of adipose tissue. Obese people have a reduced response to leptin, and in some patients the weakened response is due to a mutation in the leptin receptor gene [2, 3]. The relationship between cancer development and obesity can be partially explained by the increased leptin circulation. Leptin has been classified as a growth factor because it stimulates three key pathways well known for their role in cell development: differentiation, proliferation and activation [3]. It works through transmembrane receptors (Ob-R/LEPR). Leptin regulates a number of intracellular signaling pathways that are associated with the following molecules: Janus kinase (JAK), signal transducer and activator of transcription (STAT), phosphatidylinositol-3kinases (PI3K), protein kinase B (AKT) and mitogen-activated protein kinase (MAPK). Leptin is also closely related to several growth factors, such as: vascular endothelial growth factor (VEGF), fibroblast growth factor 21 (FGF21), and insulin-like growth factor 1 (IGF-1) [4]. In the process of carcinogenesis, leptin promotes the growth of cancer by stimulating cellular pathways that are beneficial for proliferation and acting that suppresses the apoptosis. It has been demonstrated so far that leptin escalates tumor vascularization, stimulates proliferation, migration and invasion, and suppresses apoptosis of cancer cells (Fig. 1) [4, 5].

In this literature review we examined the role of leptin in the development of individual cancers: breast cancer, colorectal cancer, prostate cancer, ovarian cancer, endometrial cancer and brain primary neoplasms: glioma and meningioma. We carried out a comprehensive literature search using the PubMed database. The range of years of searched articles was 2008-2018. The following terms were used for searching: leptin, carcinogenesis, breast 


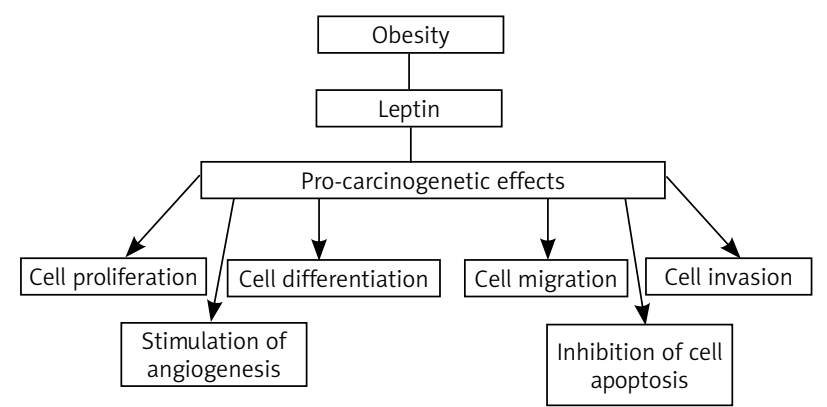

Fig. 1. Schematic representation of the pro-carcinogenic effects of leptin

cancer, colorectal cancer, prostate cancer, ovarian cancer, endometrial cancer, glioma and meningioma.

\section{The influence of leptin on the development of breast cancer}

Breast cancer is the most commonly diagnosed cancer and the most common cause of death from cancer among women around the world, with an estimated 1.7 million cases and 521,900 deaths in 2012 [6]. It has been established that overweight and obesity are important risk factors for developing breast cancer. Epidemiological studies have shown that excessive body mass significantly increases the risk of breast cancer occurrence in postmenopausal women by $20-40 \%$ [7]. The studies indicate that the leptin level is significantly associated with the occurrence of breast cancer. Higher leptin concentrations in the serum are observed in obese patients with breast cancer compared to people with normal body mass [8, 9]. Mechanisms in which obesity contributes to the development of breast cancer are complex and have not yet been fully resolved. Adipocytes constitute the highest percentage among cells that contain mammary gland tissue, and therefore they are considered as a critical type of cells in the breast cancer microenvironment [10]. They are not only close to the neoplasm cells, but also play an active role in the whole process of development, progression, metastasis and response to treatment of breast cancer [10]. Many data show a significantly higher level of leptin expression in the adipose tissue near the neoplasm, compared to the adipose tissue distant from the tumor [11]. Previous studies confirmed overexpression of leptin and Ob-R in breast tumor tissues, especially in high-grade tumors [12, 13]. Various leptin activities have been proposed to try to explain its influence on cancer progression. First, leptin activates various signaling pathways, including estrogen receptor signaling, leading to uncontrolled proliferation of the breast cancer cells $[10,14]$. Previous studies have reported that the leptin level and Ob-R expression were not only correlated with tumor size but also with receptor expression level for estrogen, as well as being significantly higher in the breast cancer patients than in healthy persons from a control group $[15,16]$. Leptin promotes angiogenesis and proinflammatory responses, as well as stimulating the proliferation of normal and neoplastic epithelial cells of the breast gland tubules [11]. Incorrect control of the cancer cells' proliferation processes is, among others, caused by HER2 receptor transactivation (human epidermal growth factor receptor 2) [11]. HER2 is overexpressed in about 30\% of breast cancer cases, and its presence indicates a more aggressive course of the disease (a negative prognosis factor) [17]. The influence of leptin on an increased proliferative activity of the breast cancer cells is also observed as a result of activation of the JAK/STAT3 and PI3K/AKT pathways $[11,16]$. Leptin acts as a cell cycle modifier, reduces the number of cells of G0/G1 phase and increases the number of breast cancer cells with the $S$ and $G 2 / M$ phases [11]. The influence of leptin on tumor progression was also found in the mechanism of apoptosis regulation. Leptin suppresses the apoptosis of breast cancer cells by increasing the expression of apoptosis inhibitors, such as Bcl-2 and $\mathrm{Bcl}-\mathrm{xl}$ antiapoptotic proteins. Finally, leptin promotes the process of angiogenesis by increasing the expression of VEGF, thereby contributing to cancer progression [11].

\section{The influence of leptin on the development of colorectal cancer}

Epidemiological data suggest that obesity is one of the risk factors for developing colorectal cancer [1]. Previous studies have shown a direct contribution of adipose tissue to the development of colorectal cancer [18-20]. The studies were conducted to investigate the expression of leptin in patients with colorectal and rectum cancer and to determine the influence of leptin on colorectal cancer cells. It was found that in patients with more advanced disease there is a statistically higher concentration of leptin in blood serum compared to patients with less advanced disease and to the control group [18]. The study results confirm that the levels of leptin and its receptor expression correlate with the tumor grading, intestinal wall invasion and distant metastases in patients [19]. Leptin has been shown to induce phosphorylation of JAK2 and extracellular signal regulated kinase (ERK), thus activating key signal transduction pathways associated with the growth of neoplasm cells [19]. In addition, it was ascertained that leptin/ $\mathrm{Ob}-\mathrm{R}$ and VEGF expression in colorectal cancer was positively correlated, resulting in accelerated angiogenesis, the degree of invasion and the presence of distant metastases in the colorectal cancer [20]. Other studies have shown that overexpression of leptin was significantly associated with the stage in the PTNM classification and the presence of lymph node metastases, distant metastases, as well as the grading and expression of phosphorylated mammalian target of rapamycin (p-mTOR), p-70S6 and p-Akt kinase [21]. In addition, the influence of leptin on the proliferation and apoptosis of the colon cancer cells was determined. The results confirmed that leptin can stimulate proliferation and suppress apoptosis of colon cancer cells by acting through the PI3K/Akt/mTOR pathway [21, 22]. Resistance to leptin associated with obesity leads to changes in gene expression and preservation of the cancer cells [23]. Studies have been carried out on the participation of microRNA (miRNA) and leptin in regulation of the expression of genes that are crucial for the carcinogenesis process in colorectal cancer cells. The experiments showed that leptin 
significantly increased miR-4443 in colorectal cancer cells, while significantly reducing the ability to invade cells, suggesting that miR-4443 acts in a way that suppresses tumor growth. Resistance to leptin can suppress this pathway and increases the risk of metastatic colorectal cancer [23].

\section{The influence of leptin on the development of prostate cancer}

There is some evidence suggesting that leptin may play a role in the development of prostate cancer. Obesity influences the progression of prostate cancer, and the adipose tissue adhering to the prostate gland is considered a potential stimulus of a progressive disease [24]. Studies show that a higher leptin level in obese men can be considered as a risk factor for prostate cancer [25]. There was confirmed significant expression of leptin receptors in prostate cancer cells, suggesting a stimulatory action of the leptin on the proliferative activity of the tested prostate cancer cell lines [26]. In addition, it was ascertained that leptin is significantly expressed in the adipose tissue adhering to the prostate gland, which is the microenvironment of the prostate cancer [27]. It has been proven that long-term exposure to leptin increased proliferation, migration and invasion of prostate cancer cells [28]. The mechanism of induction by leptin proliferation of the prostate cancer cells was suggested in a study showing that leptin affects the metabolism of estrogen and causes an increase in estrogen receptor expression [29]. Leptin also induces VEGF expression by transforming growth factors in the prostate cancer cells. This process stimulates the angiogenesis and proliferation of prostate cancer cells [30].

\section{The influence of leptin on the development of ovarian cancer}

About 230,000 cases of ovarian cancer per year are diagnosed around the world and about 140,000 women die from this disease [31]. Many factors contribute to this result, including late diagnosis, lack of highly therapeutic chemotherapy and a high degree of molecular impurity among the ovarian neoplasms. Evidence linking obesity with ovarian cancer remains unclear; however, the accumulated epidemiological data have begun to support the role of obesity in the carcinogenesis of ovarian cancer. In this respect, there were carried out studies which established the relationship between the occurrence of ovarian cancer and a high body mass index (BMI) (high obesity rate). The relative risk for ovarian cancer to increase the body weight by $5 \mathrm{~kg} / \mathrm{m}^{2}$ was 1.10 in patients who never used hormone therapy and 0.95 for women using hormone therapy [31]. Overweight and obesity have been identified as negative risk factors for ovarian cancer progression and overall survival time of patients [32]. Studies confirm the negative influence of higher concentrations of leptin in blood in obese patients with serous ovarian cancer [33]. While testing concentrations of leptin in blood serum and ascites samples collected from ovarian cancer patients with a normal BMI index and overweight, it was found that leptin concentration in blood correlates positively with the ascites fluid volume. The patients with overweight and obesity had a higher ascites fluid volume compared to people with normal BMI. Therefore, a high level of leptin in obese patients may contribute to the maintenance and survival of dormant cancer cells that remain after surgical removal (e.g. the cancer cells in ascites or abdominal cavity), which in turn increases the recurrence of the disease [33]. The mechanism of how obesity contributes to the development of ovarian cancer and affects its behavior is not fully understood. One potential explanation that has been proposed is the theory of inflammation. This conception claims that chronic exposure of the ovarian epithelium to an inflammatory stimulus triggers a malignant transformation and then favors more aggressive behavior of the neoplasm cells [34]. Excess adipose tissue and the condition of a chronic low inflammation degree characterize obesity. In this activated state, adipose cells secrete adipokines, including leptin and several inflammatory cytokines that have been associated with tumor progression and metastatic potential in other epithelial cancers, including breast, colorectal and prostate cancer [35]. In the studies there was ascertained excessive receptor expression for leptin in serous ovarian cancer cells (especially in borderline malignancy) compared to benign ovarian neoplasms. It was also confirmed that leptin significantly increases proliferation in ovarian cancer cells, but only in those with high expression of the leptin receptor [36, 37]. Furthermore, another study demonstrated a stimulatory effect of leptin on cell proliferation and an inhibitory effect on apoptosis in the human ovarian cancer cell line by up-regulating cell cycle progression genes (up-regulation of mRNAs for CCND1, CCNA2, PCNA, and E2F3 - genes responsible for inducing cell proliferation) and suppressing cell cycle inhibitor genes (down-regulation of mRNAs for P21CIP1, P27KIP1, and GADD45A - genes responsible for inhibiting proliferation). The analysis also showed that leptin promotes cell cycle progression, as demonstrated by an increased cell population in both the $S$ and G2/M phases [38]. Other studies confirmed twofold higher leptin receptor gene and protein expression in both epithelial ovarian cancer and cancer granulosa cell lines compared to non-cancer cell lines, which is associated with poor treatment prognosis $[39,40]$. In addition leptin has also been shown to stimulate the migration and invasion of ovarian cancer cells, regardless of the degree of leptin receptor expression in the cells $[36,37]$. Other studies demonstrated that the high leptin level observed in obese patients activates the PI3K/AKT/mTOR signaling pathway in ovarian cancer cells. It has been hypothesized that therapies targeting mTOR activity (caloric restriction, rapamycin, metformin and statins which suppress the mTOR pathway) should be effective against the ovarian cancer, especially in obese patients [41]. There have been conducted studies in which the pharmacological suppression of the PI3K/AKt/ mTOR pathway disrupted the malignant leptin-induced transformation of ovarian cancer cells, and it was shown that blocking the leptin receptor can radically suppress the spread of ovarian cancer cells in the peritoneal cavity, thus preventing disease progression [42]. These results identify the potential of leptin neutralization as a new strategy to support treatment of ovarian cancer [42]. 


\section{The influence of leptin on the development of endometrial cancer}

Endometrial cancer is the seventh most common cancer in women in the world. Obesity is one of the basic, if not the most important, risk factors for uterine cancer [43]. Compared to all obesity-related neoplasms, the incidence rate and mortality of endometrial cancer are the most strongly associated with an increase in BMI [43]. This is related to insulin resistance and increased estrogen production by excess adipose tissue [43]. The adipose tissue is seen as an endocrine organ synthesizing so-called adipocytokines - leptin, adiponectin, vistifin, which play a key role in the development of endometrial cancer and can be used as new markers in determining the potential risk of this disease. Chronic inflammation that accompanies obese people and those with metabolic syndrome and accompanying hyperinsulinaemia seems to have a key influence on the proliferation process of endometrial cancer cells in obese patients [43]. Many studies have shown the higher level of leptin in patients with endometrial cancer [44]. In addition, it was confirmed that there was an increased leptin level among patients with a higher stage of endometrial cancer and with a lower grade of histopathological differentiation [45]. There was also ascertained a relationship between leptin concentrations and lymph vessel infiltration and the presence of metastases to the lymph nodes [44, 45]. The results of the studies have shown that the endometrial cancer cell lines expressed higher levels of the leptin receptors as opposed to the primary non-malignant endometrial cells [45]. Overexpression of leptin and its receptors in the neoplasm cells correlated with the degree of invasion, metastasis and poor prognosis. The studies have shown the mechanism of action of leptin on the functions of endometrial cancer cells. Leptin suppresses apoptosis of endometrial cancer cells. Significant expression of leptin's receptor in the cancer cells facilitates the progression of endometrial cancer [46]. In addition, the research results indicate that leptin activates increased cell proliferation in endometrial cancer cell lines [47]. Other studies of the mechanism of endometrial cancer development focused on metabolism and estrogen synthesis, which play an important role in the formation and development of endometrial cancer [48]. The results confirmed that leptin can act as a cell growth factor. It affects the local microenvironment of the endometrial cancer by increasing the expression of aromatase P450 (P450arom) in endometrial fibroblasts, increasing the local formation of estrogen and enhancing the proliferation of endometrial cancer cells [48].

\section{The influence of leptin on the development of glioma and meningioma}

The gliomas and meningiomas are the two most common primary neoplasms of the central nervous system, representing respectively $70 \%$ and $20 \%$ of all brain tumors [49]. Risk factors for central nervous system (CNS) neoplasms are still little known. The results of a meta-analysis conducted in 2015 highlighted the significant influence of obesity as a risk factor for the development of menin- giomas and gliomas in women, as well as meningiomas in men [49]. In addition, it is proven that in patients with advanced glioma stage, obesity is an independent risk factor for early cancer progression and mortality [50]. These results were also a starting point for conducting further research on the pathophysiological relationships of the obesity mechanisms and the development of CNS neoplasms [50]. A previous study has confirmed the excessive expression of leptin and its receptor in cancer glioma cells compared to normal glioma tissue [51]. There has also been shown higher expression of leptin in high grade gliomas such as anaplastic astrocytoma or glioblastoma compared to low grade gliomas [51]. These results show a correlation with the histopathological type of the glioma and grading, resulting in more significant expression of leptin in high grade gliomas, suggesting that leptin participates in tumor progression and differentiation [52]. Searching for mechanisms linking obesity as a chronic inflammatory disease with the process of carcinogenesis, there were studies on the influence of leptin and proinflammatory factor SPLA2-IIA (proinflammatory secreted phospholipase A2 type IIA) associated with the function of astrocytoma cells. It was shown that synergistic interaction of these compounds may activate intracellular pathways participating in cancer progression and metastasis support. The SPLA2-IIA proinflammatory factor increased the expression of leptin receptors in the astrocytoma cells, whereas the mutual interaction of leptin and SPLA2-IIA caused the growth and migration of the astrocytoma cells [44]. Leptin, which increases the susceptibility of cells to inflammatory mediators, may contribute to the deterioration of the prognosis of neoplastic and neurodegenerative processes, being a potential mediator of some obesity-related complications [53]. It turns out that leptin can act as a chemo-resistant agent in the treatment of glioma. The conducted studies showed that the glioma cells with expression of leptin receptor were resistant to temozolomide (TMZ), and TMZ resistant glioblastoma cells showed overexpression of the leptin receptor. A better understanding of the mechanisms of drug resistance induced by leptin can lead to the development of more effective therapies for the treatment of glioma [54].

In the case of the evaluation of leptin receptor expression in meningiomas, there is no extensive research and the role of leptin in the development of meningioma remains unclear. The current studies did not reveal a statistically significant relationship between leptin receptor expression and the meningioma classification, but high leptin receptor expression was observed in the low malignancy grade group compared to the high malignancy grade group (46.2\% and $14.5 \%$, respectively) [55]. In addition, in patients who were found to be overweight or obese, there was significantly increased expression of the leptin receptors compared to patients who had normal body weight or underweight [55]. In other studies, it was found that leptin receptor overexpression was significantly higher in the low grade meningiomas than in the high grade meningiomas (atypical and anaplastic) $[56,57]$. The leptin receptor expression and also leptin concentration in 
the blood can be used as important prognostic markers for meningioma development $[56,57]$.

In the case of obesity, the cancer cells are constantly stimulated by pro-inflammatory cytokines and adipokines, among which leptin dominates. The studies show that leptin can affect the cancer cells through numerous phenomena, e.g. inflammation, cell proliferation, suppression of apoptosis and angiogenesis. However, leptin has very complicated mechanisms of action which require better understanding in certain types of cancer.

The authors declare no conflict of interest.

\section{References}

1. Steele CB, Thomas CC, Henley SJ, Massetti GM, Galuska DA, Agurs-Collins T, Puckett M, Richardson LC. Vital Signs: Trends in Incidence of Cancers Associated with Overweight and Obesity - United States, 2005-2014. Morb Mortal Wkly Rep 2017; 66: 1052-1058.

2. Byers T, Sedjo R. Body fatness as a cause of cancer: epidemiologic clues to biologic mechanisms. Endocr Relat Cancer 2015; 22: 125-134.

3. Nieman KM, Romero IL, Van Houten B, Lengyel E. Support tumorigenesis and metastasis. Biochim Biophys Acta 2013; 1831: 1533-1541.

4. Dutta D, Ghosh S, Pandit K, Mukhopadhyay P, Chowdhury S. Leptin and cancer: Pathogenesis and modulation. Indian J Endocrinol Metab 2012; 16 (Suppl 3): 596-S600.

5. Ray A, Cleary MP. The potential role of leptin in tumor invasion and metastasis. Cytokine Growth Factor Rev 2017; 38: 80-97.

6. Torre LA, Bray F, Siegel RL, Ferlay J, Lortet-Tieulent J, Jemal A. Global cancer statistics, 2012. CA Cancer J Clin 2015; 65: 87-108.

7. Munsell MF, Sprague BL, Berry DA, Chisholm G, Trentham-Dietz A. Body Mass Index and Breast Cancer Risk According to Postmenopausal Estrogen-Progestin Use and Hormone Receptor Status. Epidemiol Rev 2014; 36: 114-136.

8. Gui Y, Pan Q, Chen X, Xu S, Luo X, Chen L. The association between obesity related adipokines and risk of breast cancer: a meta-analysis. Oncotarget 2017; 8: 75389-75399.

9. Carroll PA, Healy L, Lysaght J, Boyle T, Reynolds JV, Kennedy MJ, Pidgeon G, Connolly EM. Influence of the metabolic syndrome on leptin and leptin receptor in breast cancer. Mol Carcinog 2011; 50: 643-651.

10. Ando S, Catalano S. The multifactorial role of leptin in driving the breast cancer microenvironment. Nat Rev Endocrinol 2011; 8: 263 275.

11. Choi J, Cha YJ, Koo J. Adipocyte biology in breast cancer: From silent bystander to active facilitator. Prog Lipid Res 2017; 21: 11-20.

12. Ishikawa M, Kitayama J, Nagawa H. Enhanced expression of leptin and leptin receptor (OB-R) in human breast cancer. Clin Cancer Res 2004; 10: 4325-4331.

13. Garofalo C, Koda M, Cascio S, Sulkowska M, Kanczuga-Koda L, Golaszewska J, Russo A, Sulkowski S, et al. Increased expression of leptin and the leptin receptor as a marker of breast cancer progression: possible role of obesity-related stimuli. Clin Cancer Res 2006; 12: 1447-1453.

14. Ray A. Adipokine leptin in obesity-related pathology of breast cancer. J Biosci 2012; 37: 289-294.

15. Romero-Figueroa MS, Garduño-García JJ, Duarte-Mote J, Matute-González G, Gómez-Villanueva A, De la Cruz-Vargas J. Insulin and leptin levels in obese patients with and without breast cancer. Clin Breast Cancer 2013; 13: 482-485.

16. Jardé T, Perrier S, Vasson MP, Caldefie-Chézet F. Molecular mechanisms of leptin and adiponectin in breast cancer. Eur J Cancer 2011; 47: 33-43.

17. Ray A. Tumor-linked HER2 expression: association with obesity and lipid-related microenvironment. Horm Mol Biol Clin Investig 2017; 32: pii: /j/hmbci.2017.32.issue-3/hmbci-2017-0020/hmbci-2017-0020.xm.
18. Yoon KW, Park SY, Kim JY, et al. Leptin-induced adhesion and invasion in colorectal cancer cell lines. Oncol Rep 2014; 31: 2493-2498.

19. Liu H, Wan D, Pan Z, Cao L, Wu X, Lu Z, Kang T. Expression and Biological Significance of Leptin, Leptin Receptor, VEGF, and CD34 in Colorectal Carcinoma. Cell Biochem Biophys 2011; 60: 241-244.

20. Milosevic VS, Vukmirovic FC, Krstic MS, Zindovic MM, Lj Stojanovic $D$, Jancic SA. Involvement of leptin receptors expression in proliferation and neoangiogenesis in colorectal carcinoma. J BUON 2015; 20: 100-108.

21. Wang D, Chen J, Chen H, Duan Z, Xu Q, Wei M, Wang L, Zhong M. Leptin regulates proliferation and apoptosis of colorectal carcinoma through PI3K/Akt/mTOR signalling pathway. J Biosci 2012; 37: 91-101.

22. Riondino S, Roselli M, Palmirotta R, Della-Morte D, Ferroni P, Guadagni F. Obesity and colorectal cancer: role of adipokines in tumor initiation and progression. World J Gastroenterol 2014; 20: 51775190.

23. Meerson A, Yehuda H. Leptin and insulin up-regulate miR-4443 to suppress NCOA1 and TRAF4, and decrease the invasiveness of human colon cancer cells. BMC Cancer 2016; 16: 882.

24. Xu L, Shen M, Chen X, et al. Adipocytes affect castration-resistant prostate cancer cells to develop the resistance to cytotoxic action of NK cells with alterations of PD-L1/NKG2D ligand levels in tumor cells. Prostate 2018; 78: 353-364.

25. Kang M, Byun SS, Lee SE, Hong SK. Clinical Significance of Serum Adipokines according to Body Mass Index in Patients with Clinically Localized Prostate Cancer Undergoing Radical Prostatectomy. World J Mens Health 2018; 36: 57-65.

26. Szyszka M, Tyczewska M, Milecka P, Jopek K, Celichowski P, Malendowicz LK, Rucinski M. Effects of leptin on leptin receptor isoform expression and proliferative activity in human normal prostate and prostate cancer cell lines. Oncol Rep 2018; 39: 182-192.

27. Zhang Q, Sun LJ, Yang ZG, Zhang GM, Huo RC. Influence of adipocytokines in periprostatic adipose tissue on prostate cancer aggressiveness. Cytokine 2016; 85: 148-156.

28. Noda T, Kikugawa T, Tanji N, Miura N, Asai S, Higashiyama S, Yokoyama $M$. Long term exposure to leptin enhances the growth of prostate cancer cells. Int J Oncol 2015; 46: 1535-42.

29. Habib CN, Al-Abd AM, Tolba MF, Khalifa AE, Khedr A, Mosli HA, Abdel-Naim AB. Leptin influences estrogen metabolism and accelerates prostate cell proliferation. Life Sci 2015; 121: 10-15.

30. Alshaker H, Sacco K, Alfraidi A, Muhammad A, Winkler M, Pchejetski D. Leptin signalling, obesity and prostate cancer: molecular and clinical perspective on the old dilemma. Oncotarget 2015; 6: 35556-35563.

31. Collaborative Group on Epidemiological Studies of Ovarian Cancer Ovarian cancer and body size: individual participant meta-analysis including 25,157 women with ovarian cancer from 47 epidemiological studies. PLoS Med 2012; 9: e1001200.

32. Protani MM, Nagle CM, Webb PM. Obesity and ovarian cancer survival: a systematic review and meta-analysis. Cancer Prev Res 2012; 5: 901-910.

33. Kato S, Abarzua-Catalan L, Trigo C, et al. Leptin stimulates migration and invasion and maintains cancer stem-like properties in ovarian cancer cells: an explanation for poor outcomes in obese women. Oncotarget 2015; 6: 21100-21119.

34. Maccio A, Madeddu C. Inflammation and ovarian cancer. Cytokine 2012; 58: 133-147.

35. Nieman KM, Romero IL, Van Houten B, Lengyel E. Adipose tissue and adipocytes support tumorigenesis and metastasis. Biochim Biophys Acta 2013; 1831: 1533-1541.

36. Ghasemi A, Hashemy SI, Aghaei M, Panjehpour M. RhoA/ROCK pathway mediates leptin-induced UPA expression to promote cell invasion in ovarian cancer cells. Cell Signal 2017; 32: 104-114.

37. Kumar J, Fang H, McCulloch DR, Crowley T, Ward AC. Leptin receptor signaling via Janus kinase 2/Signal transducer and activator of transcription 3 impacts on ovarian cancer cell phenotypes. Oncotarget 2017; 8: 93530-93540.

38. Ptak A, Kolaczkowska E, Gregoraszczuk EL. Leptin promotes epithelial ovarian cancer cell growth by up-regulating genes and proteins responsible for inducing cell proliferation as well as 
down-regulating proapoptotic genes and proteins in apoptotic pathways. Endocrine 2013; 43: 394-403.

39. Fiedor E, Gregoraszczuk EL. Superactive human leptin antagonist (SHLA), triple Lan1 and quadruple Lan2 leptin mutein as a promising treatment for human folliculoma. Cancer Chemother Pharmacol 2017; 80: 815-827.

40. Fiedor E, Gregoraszczuk EL. The molecular mechanism of action of superactive human leptin antagonist (SHLA) and quadruple leptin mutein Lan-2 on human ovarian epithelial cell lines. Cancer Chemother Pharmacol 2016; 78: 611-622.

41. Mabuchi S, Kuroda H, Takahashi R, Sasano T. The PI3K/AKT/mTOR pathway as a therapeutic target in ovarian cancer. Gynecol Oncol 2015; 137: 173-179.

42. Wei X, Liu Y, Gong C, et al. Targeting Leptin as a Therapeutic Strategy against Ovarian Cancer Peritoneal Metastasis. Anticancer Agents Med Chem 2017; 17: 1093-1101.

43. Daley-Brown D, Oprea-llies GM, Lee R, Pattillo R, Gonzalez-Perez RR. Molecular cues on obesity signals, tumor markers and endometrial cancer. Horm Mol Biol Clin Investig 2015; 21: 89-106.

44. Cymbaluk-Płoska A, Chudecka-Głaz A, Jagodzińska A, Pius-Sadowska E, Sompolska-Rzechuła A, Machaliński B, Menkiszak J. Evaluation of biologically active substances promoting the development of or protecting against endometrial cancer. Onco Targets Ther 2018; 11: 1363-1372.

45. Zhang Y, Liu L, Li C, Ai H. Correlation analysis between the expressions of leptin and its receptor (ObR) and clinicopathology in endometrial cancer. Cancer Biomark 2014; 14: 353-359.

46. Zhou X, Li H, Chai Y, Liu Z. Leptin Inhibits the Apoptosis of Endometrial Carcinoma Cells Through Activation of the Nuclear Facto $\kappa B$-inducing Kinase/IкB Kinase Pathway. Int J Gynecol Cancer 2015; 25: 770-778.

47. Gao J, Tian J, Ly Y. Leptin induces functional activation of cyclooxygenase-2 through JAK2/STAT3, MAPK/ERK and PI3K/AKT pathways in human endometrial cancer cells. Cancer Sci 2009; 100: 389-395.

48. Liu L, Wang L, Zheng J, Tang G. Leptin promotes human endometrial carcinoma cell proliferation by enhancing aromatase (P450arom) expression and estradiol formation. Eur J Obstet Gynecol Reprod Biol 2013; 170: 198-201.

49. Sergentanis TN, Tsivgoulis G, Perlepe C, Ntanasis-Stathopoulos I, Tzanninis IG, Sergentanis IN, Psaltopoulou T. Obesity and Risk for Brain/CNS Tumors, Gliomas and Meningiomas: A Meta-Analysis. PLoS One 2015; 10: e0136974.

50. Chambless LB, Parker SL, Hassam-Malani L, McGirt MJ, Thompson RC. Type 2 diabetes mellitus and obesity are independent risk factors for poor outcome in patients with high-grade glioma. J Neurooncol 2012; 106: 383-389.

51. Riolfi M, Ferla R, Valle LD, et al. Leptin and its receptor are overexpressed in brain tumors and correlate with the degree of malignancy. Brain Pathol 2010; 20: 481-489.

52. Lawrence JE, Cook NJ, Rovin RA, Winn RJ. Leptin promotes glioblastoma. Neurol Res Int 2012; 2012: 870807.

53. Martín R, Cordova C, Gutiérrez B, Hernández M, Nieto ML. A dangerous liaison: Leptin and SPLA2-IIA join forces to induce proliferation and migration of astrocytoma cells. PLoS One 2017; 12: e0170675.

54. Han G, Wang L, Zhao W, et al. High expression of leptin receptor leads to temozolomide resistance with exhibiting stem/progenitor cell features in glioblastoma. Cell Cycle 2013; 12: 3833-3840.

55. Rutkowski R, Reszec J, Hermanowicz A, Chrzanowski R, Lyson T, Mariak Z, Chyczewski L. Correlation of leptin receptor expression with BMI in differential grades of human meningiomas. Oncol Lett 2016; 11: 2515-2519.

56. Menghi F, Orzan FN, Eoli M, et al. DNA microarray analysis identifies CKS2 and LEPR as potential markers of meningioma recurrence. Oncologist 2011; 16: 1440-1450.

57. Schmidt M, Mock A, Jungk C, et al. Transcriptomic analysis of aggressive meningiomas identifies PTTG1 and LEPR as prognostic biomarkers independent of WHO grade. Oncotarget 2016; 7: 14551-14568.

\section{Address for correspondence}

\section{Patrycja Modzelewska}

Department of Integrated Medical Care Department of Medical Pathomorphology Medical University of Bialystok

1 Jana Kilińskiego St.

15-089 Bialystok, Poland

e-mail: pati31802@wp.pl

Submitted: 10.09 .2018

Accepted: 29.04.2019 